\section{(O) OPEN ACCESS}

\title{
The dual impact of antiretroviral therapy and sexual behaviour changes on HIV epidemiologic trends in Uganda: a modelling study
}

\author{
Leigh Anne Shafer, ${ }^{1,2}$ Rebecca N Nsubuga, ${ }^{2}$ Ruth Chapman, ${ }^{3}$ Katie O'Brien, ${ }^{3}$ \\ Billy N Mayanja, ${ }^{2}$ Richard G White ${ }^{3}$
}

- Additional material is published online only. To view please visit the journal online (http://dx.doi.org/10.1136/ sextrans-2013-051219).

1 Department of Internal Medicine, University of Manitoba, Winnipeg, Manitoba, Canada

${ }^{2}$ Medical Research Council Unit on AIDS/Uganda Virus Research Institute, Entebbe, Uganda

${ }^{3}$ London School of Hygiene and Tropical Medicine, London, UK

\section{Correspondence to} Dr Leigh Anne Shafer, Department of Internal Medicine, University of Manitoba, GF335, 810 Sherbrook Avenue, Winnipeg, Manitoba, Canada R3E 3P5; leighanne.shafer@med. umanitoba.ca

Received 4 June 2013 Revised 29 January 2014 Accepted 2 February 2014 Published Online First 24 February 2014

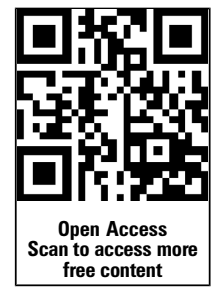

\section{ABSTRACT}

Objectives Antiretroviral therapy (ART) availability in a population may influence risky sexual behaviour. We examine the potential impact of ART on the HIV epidemic, incorporating evidence for the impact that ART may have on risky sexual behaviour.

Methods A mathematical model, parameterised using site-specific data from Uganda and worldwide literature review, was used to examine the likely impact of ART on HIV epidemiologic trends. We varied assumptions about rates of initiating ART, and changes in sexual partner turnover rates.

Results Modelling suggests that ART will reduce HIV incidence over 20 years, and increase prevalence. Even in the optimistic scenario of ART enrollment beginning after just five months of infection (in HIV stage 2), prevalence is estimated to rise from a baseline of $10.5 \%$ and $8.3 \%$ among women and men, respectively, to at least $12.1 \%$ and $10.2 \%$, respectively. It will rise further if sexual disinhibition occurs or infectiousness while on ART is slightly higher ( $2 \%$ female to male, rather than $0.5 \%$ ). The conditions required for ART to reduce prevalence over this period are likely too extreme to be achievable. For example, if ART enrolment begins in HIV stage 1 (within the first 5 months of infection), and if risky sexual behaviour does not increase, then 3 of our 11 top fitting results estimate a potential drop in HIV prevalence by 2025. If sexual risk taking rises, it will have a large additional impact on expected HIV prevalence. Prevalence will rise despite incidence falling, because ART extends life expectancy.

Conclusions HIV prevalence will rise. Even small increases in partner turnover rates will lead to an additional substantial increase in HIV prevalence. Policy makers are urged to continue HIV prevention activities, including promoting sex education, and to be prepared for a higher than previously suggested number of HIV infected people in need of treatment.

\section{INTRODUCTION}

Antiretroviral therapy (ART) use is now widespread in sub-Saharan Africa. ${ }^{1}$ Recent evidence suggests that ART may reduce population-level incidence of HIV. $^{2}$ Modelling studies have also investigated the potential impact of ART. ${ }^{3-12}$ These studies have reached varying conclusions. Treatment prolongs the lives of those infected, so some studies have suggested increased prevalence due to ART. ${ }^{6} 913$ However, it has also been suggested that frequent testing and early ART enrolment could reduce HIV prevalence, ${ }^{7} 810$ and may even eradicate the epidemic. $^{10}{ }^{14} \mathrm{HIV}$-infected individuals on ART are less infectious than those not on ART. ${ }^{15-17}$ The differing conclusions are largely dependent on assumptions, and may be particularly sensitive to assumptions about sexual behaviour. ${ }^{12}$ ART may lead to sexual disinhibition as people feel that they may live a long life with HIV.

The overall impact that ART may have on the HIV epidemic sometimes incorporates the potential impact of ART on sexual behaviour. ${ }^{6} 10$ However, the ranges of potential behaviour change are often not based on data, and do not include the impact that ART availability may have on behaviour among HIV uninfected people. Previous studies have provided contrasting results regarding the impact of ART on sexual behaviour. ${ }^{18-20}$

Previously, we examined self-reported evidence for changing sexual behaviour after the introduction of ART in a rural Ugandan cohort in 2004. ${ }^{21}$ We found evidence that risky behaviour, particularly partner turnover rates, may rise among HIV uninfected people in response to the availability of ART.

In the Ugandan cohort mentioned above, HIV prevalence rose from $6.87 \%$ in 2004 , the year that ART roll-out began, to $8.75 \%$ by 2012 . However, in this cohort, most people do not begin ART treatment until HIV stage 3.

Here, through mathematical modelling, we assess the plausible impact of ART on future HIV prevalence and incidence under different scenarios of rate of ART enrolment. As some have postulated that ART may be a means of eradicating HIV, ${ }^{10} 14$ our objective is to assess whether ART could conceivably reduce or eliminate the HIV epidemic, under extremely optimistic conditions. We examine impact in a range of scenarios with varying assumptions about the average time from HIV infection until ART enrolment, infectiousness while on ART, and sexual behaviour modification. Uniquely in this study, we incorporated evidence-driven potential sexual behaviour change due to ART among people infected with HIV, as well as due to the availability of ART among those uninfected with HIV.

\section{METHODS}

In 1989, the Medical Research Council established a general population cohort (GPC) in rural Uganda. ${ }^{22-24}$ Participants are serotested annually for HIV. We present HIV prevalence estimates from 
this cohort, following estimation methods described previously. $^{25} 26$ We fit our model to prevalence data through 2004, which is the last year in which the impact of ART on epidemiologic trends would not yet have been felt in this population.

\section{Mathematical model}

The compartmental mathematical model is stratified by age, sex and sexual activity group (figure 1). Details are online (see online supplementary appendix). Results are based on simulations with average durations in HIV stages $1-4$ of 5 months, 7 years, 18 months and 10 months.

Upon ART enrolment in the model, infectiousness drops. At a rate of $0.5 /$ year, those who have not yet died move into a compartment with mortality rate equivalent to background mortality. It is assumed that after surviving the initial ART period, life expectancy on ART is the same as that among HIV uninfected people.

\section{Baseline scenario}

The baseline scenario was fit to data from Uganda and literature reviews. We fit to empirically estimated HIV prevalence by gender among 15-54-year-olds from 1991 to 2004, before ART can have impacted prevalence. To fit, we ran 750000 simulations with varying parameter values (see online supplementary table S1). Parameters include behaviour change before the introduction of ART, consistent with evidence suggesting that risky behaviour declined during the 1990s. ${ }^{27}$ The parameter set with the highest goodness of fit between model and empirically estimated prevalence was considered the best fitting.
Table 1 ART Impact Scenarios

\begin{tabular}{|c|c|c|c|}
\hline Scenario & $\begin{array}{l}\text { Probability of HIV } \\
\text { transmission while } \\
\text { on ART (per } \\
\text { partnership) }\end{array}$ & $\begin{array}{l}\text { Earliest HIV stage of } \\
\text { ART enrolment }\end{array}$ & $\begin{array}{l}\text { Modelled change } \\
\text { in sexual partner } \\
\text { turnover? }\end{array}$ \\
\hline 1 & $\begin{array}{l}0.5 \% \mathrm{~F}->\mathrm{M}, \\
1.0 \% \mathrm{M}->\mathrm{F}\end{array}$ & $\begin{array}{l}3 \text { (rate of initiation } \\
\text { varies) }\end{array}$ & No \\
\hline 2 & $\begin{array}{l}2.0 \% \mathrm{~F}->\mathrm{M}, \\
4.0 \% \mathrm{M}->\mathrm{F}\end{array}$ & $\begin{array}{l}3 \text { (rate of initiation } \\
\text { varies) }\end{array}$ & No \\
\hline 3 & $\begin{array}{l}0.5 \% \mathrm{~F}->\mathrm{M}, \\
1.0 \% \mathrm{M}->\mathrm{F}\end{array}$ & $\begin{array}{l}1 \text { (rate of initiation } \\
\text { varies) }\end{array}$ & No \\
\hline 4 & $\begin{array}{l}2.0 \% \mathrm{~F}->\mathrm{M}, \\
4.0 \% \mathrm{M}->\mathrm{F}\end{array}$ & $\begin{array}{l}1 \text { (rate of initiation } \\
\text { varies) }\end{array}$ & No \\
\hline 5 & $\begin{array}{l}0.5 \% \mathrm{~F}->\mathrm{M}, \\
1.0 \% \mathrm{M}->\mathrm{F}\end{array}$ & $\begin{array}{l}2 \text { (rate of } \\
\text { initiation=0.9/year) }\end{array}$ & Yes \\
\hline 6 & $\begin{array}{l}2.0 \% \mathrm{~F}->\mathrm{M}, \\
4.0 \% \mathrm{M}->\mathrm{F}\end{array}$ & $\begin{array}{l}2 \text { (rate of } \\
\text { initiation }=0.9 / \text { year) }\end{array}$ & Yes \\
\hline
\end{tabular}

\section{ART impact scenarios}

ART introduction was simulated in 2004. We examined six ART scenarios (table 1).

Within each of the first four scenarios, we varied the annual rate of ART enrolment once the respective HIV stage was reached, from 0.0 (baseline-no ART) to 1.0. In these scenarios, we assumed that partnership turnover rates did not change after ART introduction. We examined two scenarios in which ART enrolment may begin as early as HIV stage 1, and two in which ART enrolment may begin in HIV stage 3 . We present the

\section{(Enclosed in parentheses are the symbols used in the differential equations.)}

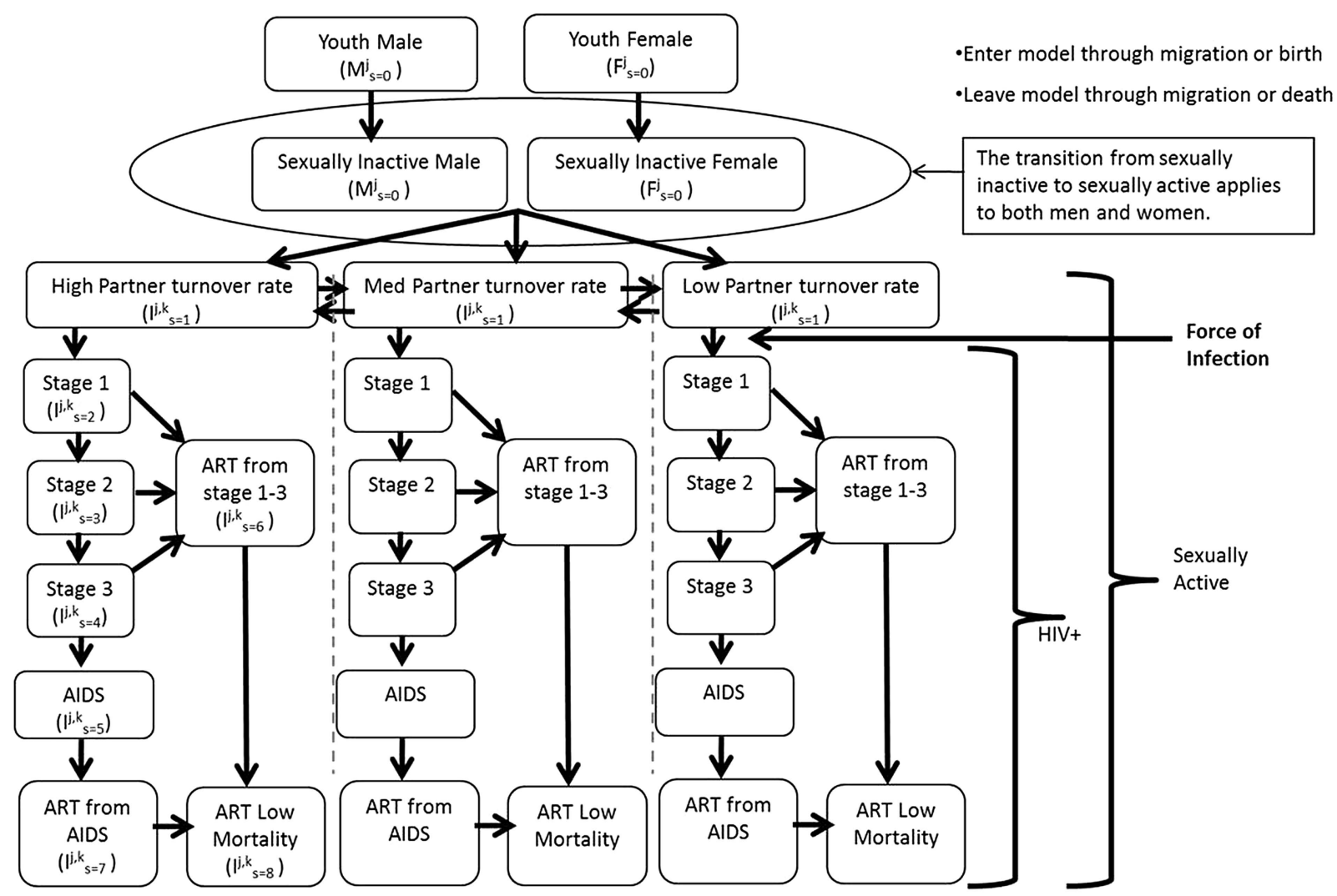

Figure 1 Compartmental Mathematical Model (Enclosed in parentheses are the symbols used in the differential equations.) 
scenarios of ART enrolment beginning in stages 1 and 3 as these are boundaries. It may be unlikely to expect ART enrolment in stage 1 often enough to impact the epidemic, but we present this scenario as it represents the upper bound of the impact that ART could possibly have on the HIV epidemic.

When examining the impact of increasing partner turnover rates in response to ART availability, it was assumed that ART enrolment may begin as early as HIV stage 2, and that the enrolment rate was $0.9 /$ year. The percent increase in partner turnover rate beginning in 2004 varied from $0.0 \%$ (no change) to $50.0 \%$. HIV stage 2 represents a reasonable yet aggressive timing for ART enrolment.

When HIV transmission while on ART was $1 \%$ male to female $(0.5 \%$ female to male), this represented a percent reduction in transmission probability in our best fitting scenario among men ranging from $85.1 \%$ to $96.6 \%$, and among women ranging from $90.9 \%$ to $97.6 \%$. The percent reduction depended on the stage from which ART was initiated, and the probability of transmission from the respective stage before ART enrolment.

\section{Goodness-of-fit measures and sensitivity analysis}

We assessed the sensitivity of results to parameter value set selection by summarising the results from multiple good fitting parameter sets: We found the best fitting parameter values using three goodness-of-fit (GoF) measures: a sum of squares, a $\chi^{2},{ }^{28}$ and maximum likelihood. ${ }^{29}{ }^{30}$ We used three GoF measures because some readers may be more confident in results of one or another of the measures, but the three measures produced significantly overlapping best fits. ${ }^{2}$ We compared results using the first through seventh best fitting parameter sets.

Of the 21 sets of parameter values, 10 were repeats. That is, five of the seven best fitting sets found when maximising the likelihood function were the same as those found when

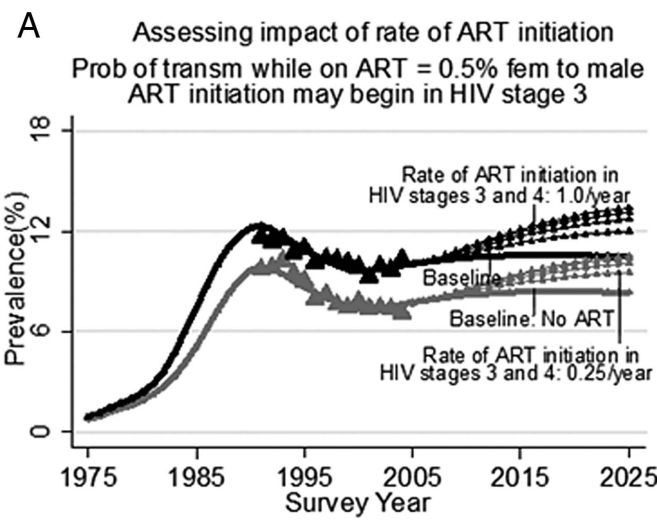

C Assessing impact of rate of ART initiation Prob of transm while on ART $=0.5 \%$ fem to male ART initiation may begin as early as HIV stage 1

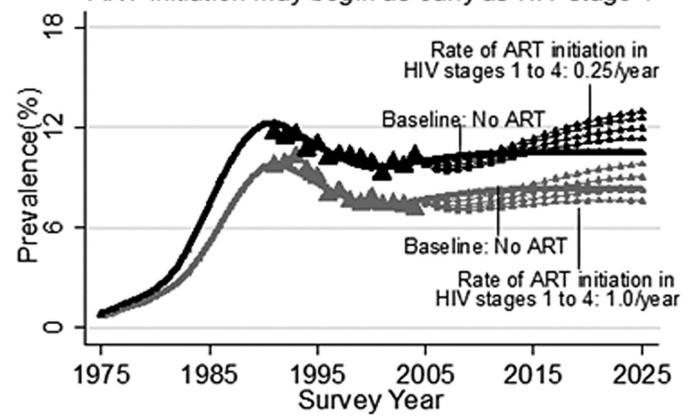

E Assessing impact of increased partner tumover rat€ Prob of transm while on ART $=0.5 \%$ fem to male ART initiation begins in stage 2 at a rate of 0.9 /year

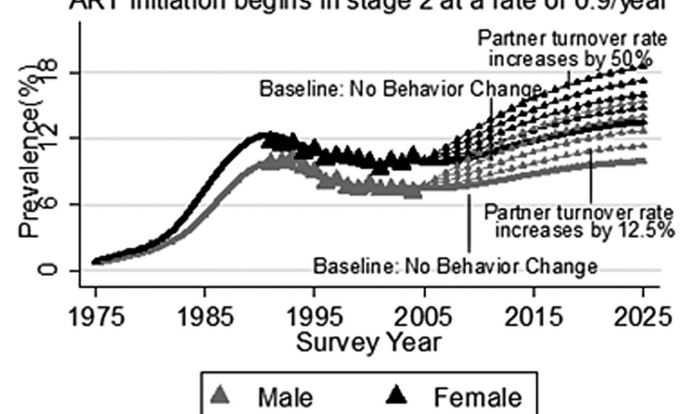

B Assessing impact of rate of ART initiation

Prob of transmission while on ART $=2.0 \%$ female to male ART initiation may begin in HIV stage 3

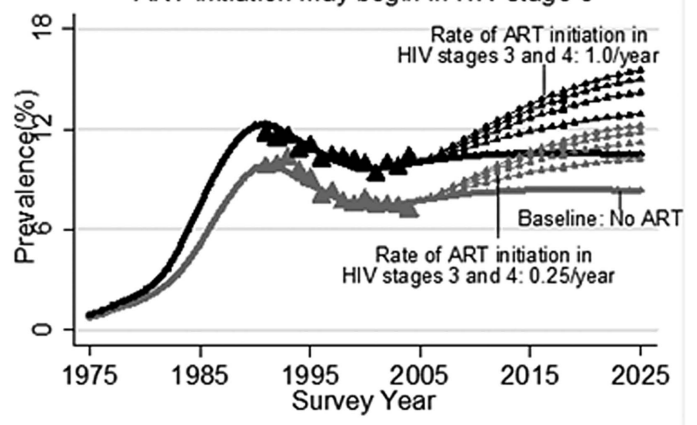

D Assessing impact of rate of ART initiation Prob of transmission while on ART $=2.0 \%$ female to male

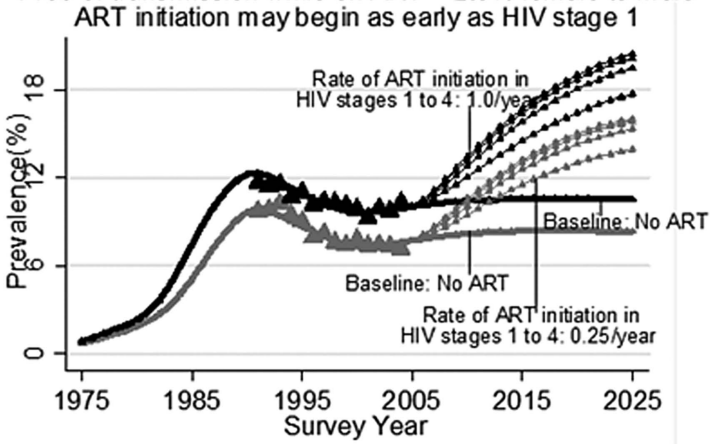

F Assessing impact of increased partner tumover rate Prob of transmission while on ART $=2.0 \%$ female to male ART initiation begins in stage 2 at a rate of 0.9 /year

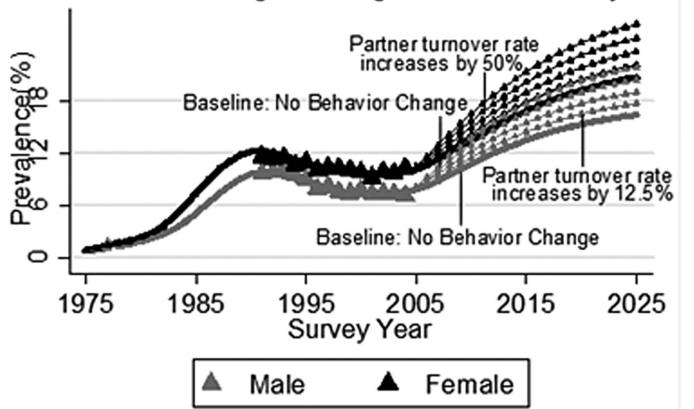

Figure 2 Sensitivity Analyses of Dual Impact of antiretroviral therapy (ART) and Potential Behavior Change on HIV Prevalence. 
minimising the sum of squared differences, and five of those found under the $\chi^{2}$ GoF had also been found under either the sum of squares or maximum likelihood GoF. We used the remaining 11 parameter sets to assess robustness of model results.

\section{RESULTS}

In the best fit, HIV transmission probability per partnership and stage is shown in online supplementary table S1.

\section{Prevalence}

Among the 11 best fitting simulations, we obtained modelestimated prevalence continuously from 1975 to 2025. The mean of these model estimates is presented in figure 2 , for a range of different scenarios. Panels $\mathrm{A}$ and $\mathrm{B}$ correspond to scenario sets 1 and 2, described in methods. With no ART, HIV prevalence reaches $10.5 \%$ among women and $8.3 \%$ among men by 2025 . In panel A, if the rate of ART enrolment beginning in stage 3 is 0.25 year, then predicted HIV prevalence rises to $12.0 \%$ among women and $9.5 \%$ among men by 2025 . Panel B portrays a scenario set in which the drop in the probability of HIV transmission while on ART is less than that in panel A. In this case, under an otherwise similar scenario to that just described, prevalence rises to $12.9 \%$ among women and $10.2 \%$ among men by 2025 . The increase in HIV prevalence is more pronounced in these scenarios if people begin ART at a faster rate. If ART enrolment beginning in HIV stage 3 is 1.0/year, then HIV prevalence may rise to $15.5 \%$ among women and $12.2 \%$ among men by 2025 (panel B).

In scenario set 3 , people initiate ART in HIV stage 1 , and there is no behaviour change in response to ART availability. This is the only one among the range of scenarios examined in which the average of our 11 best fitting prevalence estimates indicates that HIV prevalence may initially fall after ART introduction (figure 2C). By 10 years after ART is introduced, among men, HIV prevalence is higher if the rate of ART enrolment is less than 0.50 year than it would have been in the absence of ART, and among women it is higher regardless of ART enrolment rates. However, among men, if the rate of ART enrolment is greater than 0.50/year, and ART enrolment begins in HIV stage 1, then HIV prevalence may permanently decline, relative to what it is in the absence of ART. If the rate of ART enrolment is 1.0 /year, HIV prevalence falls to $10.5 \%$ among women and $7.4 \%$ among men by 2015 , before rising to $11.4 \%$ among women by 2025 and remaining level among men. Panel D (scenario set 4 ) portrays a similar scenario set, except that the drop in probability of HIV transmission while on ART is less. In this case, the impact of ART is to increase HIV prevalence dramatically, regardless of the rate at which people initiate ART.

We next examined the impact that ART may have on HIV prevalence if partner turnover rates increase. The scenarios depicted in figure 2E,F, are described as scenario sets 5 and 6 in methods. In panel E, a $12.5 \%$ increase in partner turnover rate results in rising HIV prevalence up to 20 years after ART is introduced. By 2025, prevalence reaches $17.7 \%$ among women and $13.9 \%$ among men, contrasting with $12.1 \%$ (women) and $10.2 \%$ (men) without behaviour change. If ART only reduces HIV transmission probability per partnership to $2 \%$ female to male (panel F), then prevalence is higher, but the relative impact of higher partner turnover rates is similar to what it is under the scenario in which ART reduces HIV transmission probability to $0.5 \%$ female to male.

As anticipated, we estimated increasing HIV prevalence in the presence of ART even when most of the HIV infected are on ART. In figure 2EF, the rate of initiating ART was $0.9 /$ year, beginning in stage 2 . In this case, the percent of HIV infected who are on ART reaches almost $90 \%$ by 20 years after the introduction of ART in the population.

\section{Sensitivity analysis}

Multiple sets of parameter values produced model estimates that fitted the empirically estimated prevalence. Without ART, the 11 sets of parameter values that most closely fit our data estimated similar HIV epidemics (figure 3).

By 2025, all 11 sets of parameter values estimated that ART would result in increased HIV prevalence, if ART enrolment begins in HIV stage 3 (table 2, scenario 1). The smallest increase in prevalence would occur if the probability of HIV transmission per partnership female to male was low at $0.5 \%$, and the rate of ART enrolment was also low at 0.25 year. In this case, by 2025 , the mean increase in prevalence was $14.6 \%$ (table 2 , scenario 1). If either the rate at which people begin ART treatment or the probability of HIV transmission while on treatment rises, then all 11 parameter sets estimated an even greater rise in HIV prevalence than under the scenario just described.
Figure 3 Prevalence by Gender-Age 15-54 Markers-Uganda Cohort Data, Lines-Model.

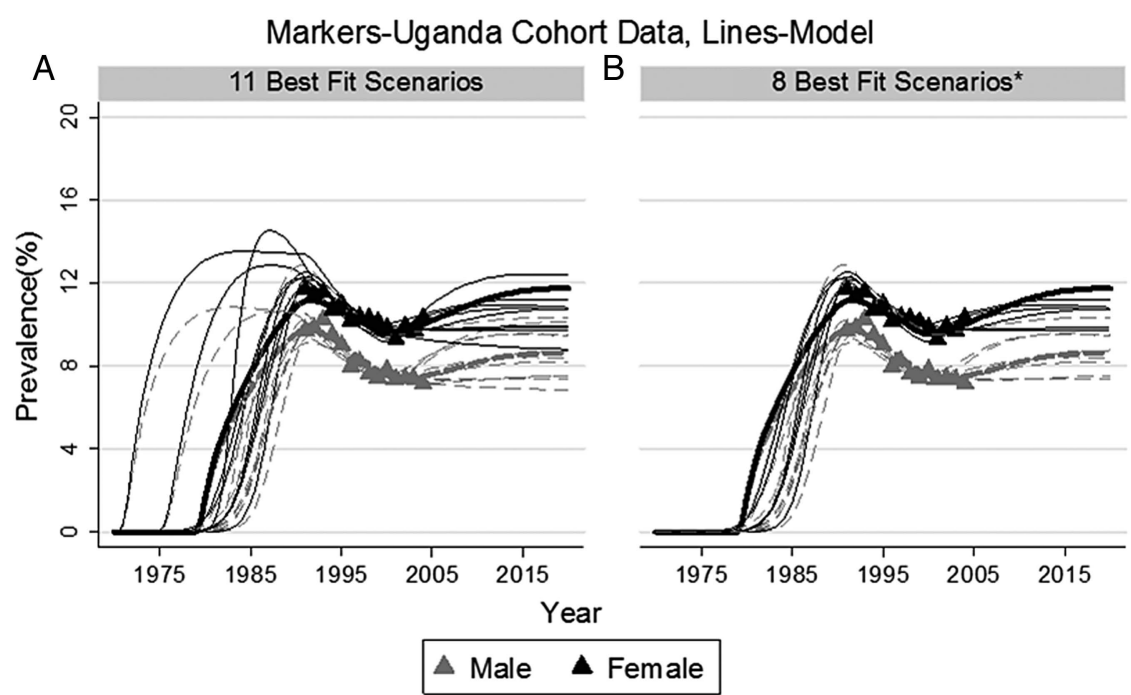

*In Panel B, the 3 scenarios estimating early HIV prevalence peaks were removed. 
Table 2 Percent increase in HIV prevalence forecasted by 11 'best fit' parameter value sets, under scenarios depicted in figure 2 (\% increase compared to scenario of no ART or no behaviour change)

\begin{tabular}{|c|c|c|c|c|c|c|c|c|c|c|c|c|c|}
\hline \multirow[b]{2}{*}{ Scenario } & \multirow[b]{2}{*}{ Year } & \multicolumn{11}{|c|}{ Modelled parameter value set } & \multirow[b]{2}{*}{ Mean (SD) } \\
\hline & & 1 & 2 & $3^{*}$ & 4 & 5 & 6 & 7 & 8 & 9 & $10^{*}$ & $11^{*}$ & \\
\hline \multicolumn{14}{|c|}{ Probability of transmission on ART $=0.5 \%$ female to male. No ART enrolment in HIV stages 1 or 2 (figure $2 \mathrm{~A}$ ) } \\
\hline \multicolumn{14}{|c|}{ Rate of ART init starting stage 3} \\
\hline \multirow[t]{2}{*}{$0.25 /$ year } & 2015 & 1.3 & 11.2 & 0.4 & -0.4 & 7.4 & 6.0 & 13.0 & 10.2 & 10.6 & 9.3 & 12.7 & $7.4(4.9)$ \\
\hline & 2025 & 6.5 & 19.3 & 5.5 & 4.5 & 14.3 & 14.2 & 20.7 & 19.4 & 18.4 & 16.9 & 21.4 & $14.6(6.3)$ \\
\hline \multirow[t]{2}{*}{ 1.00/year } & 2015 & 1.8 & 23.4 & -0.2 & -3.7 & 14.4 & 11.5 & 28.6 & 21.8 & 23.2 & 20.2 & 27.9 & $15.4(11.5)$ \\
\hline & 2025 & 9.9 & 39.0 & 7.9 & 1.6 & 26.8 & 27.2 & 43.7 & 39.5 & 38.2 & 33.9 & 45.0 & $28.4(15.4)$ \\
\hline \multicolumn{14}{|c|}{ Probability of transmission on ART=2.0\% female to male. No ART enrolment in HIV stages 1 or 2 (figure $2 \mathrm{~B}$ ) } \\
\hline \multicolumn{14}{|c|}{ Rate of ART init starting stage 3} \\
\hline \multirow[t]{2}{*}{$0.25 /$ year } & 2015 & 6.6 & 14.8 & 4.9 & 2.5 & 15.5 & 14.8 & 17.8 & 17.6 & 16.2 & 16.8 & 15.0 & $13.0(5.5)$ \\
\hline & 2025 & 16.7 & 24.1 & 13.9 & 10.4 & 25.8 & 27.1 & 27.0 & 31.5 & 26.2 & 31.2 & 24.7 & $23.5(6.9)$ \\
\hline \multirow[t]{2}{*}{ 1.00/year } & 2015 & 14.2 & 32.6 & 10.6 & 3.3 & 34.7 & 32.8 & 39.7 & 39.1 & 36.1 & 36.8 & 33.6 & $28.5(12.7)$ \\
\hline & 2025 & 32.9 & 50.9 & 27.5 & 15.5 & 55.5 & 57.1 & 58.8 & 66.2 & 56.4 & 64.8 & 53.2 & $49.0(16.3)$ \\
\hline \multicolumn{14}{|c|}{ Probability of transmission on ART $=0.5 \%$ female to male. ART enrolment begins in HIV stage 1 (figure $2 \mathrm{C}$ ) } \\
\hline \multicolumn{14}{|c|}{ Rate of ART init } \\
\hline \multirow[t]{2}{*}{ 0.25/year } & 2015 & -10.7 & 21.9 & -10.7 & -19.0 & 13.4 & 14.0 & 21.1 & 15.6 & 21.8 & 2.7 & 23.3 & $8.5(15.4)$ \\
\hline & 2025 & -4.1 & 44.7 & -4.3 & -21.5 & 30.1 & 37.5 & 42.3 & 39.4 & 44.2 & 14.4 & 49.2 & $24.7(24.5)$ \\
\hline \multirow[t]{2}{*}{ 1.00/year } & 2015 & -30.9 & 17.1 & -25.2 & -33.6 & 6.7 & 10.9 & 17.2 & 7.7 & 24.5 & -3.9 & 18.8 & $0.8(21.2)$ \\
\hline & 2025 & -41.1 & 35.5 & -25.5 & -46.8 & 12.0 & 27.4 & 32.2 & 21.6 & 47.5 & 1.2 & 43.7 & $9.8(33.6)$ \\
\hline \multicolumn{14}{|c|}{ Probability of transmission on ART=2.0\% fem ale to male. ART enrolment begins in HIV stage 1 (figure 2D) } \\
\hline \multicolumn{14}{|c|}{ Rate of ART init } \\
\hline \multirow[t]{2}{*}{ 0.25/year } & 2015 & 16.0 & 44.2 & 12.2 & -4.5 & 61.0 & 58.8 & 50.4 & 56.0 & 53.3 & 36.0 & 39.7 & $38.4(21.6)$ \\
\hline & 2025 & 42.7 & 72.8 & 36.5 & 6.2 & 98.5 & 102.0 & 81.2 & 98.7 & 87.9 & 74.1 & 71.0 & $70.1(30.1)$ \\
\hline \multirow[t]{2}{*}{ 1.00/year } & 2015 & 17.2 & 69.9 & 14.7 & -12.9 & 102.2 & 96.6 & 80.0 & 89.3 & 85.7 & 52.2 & 61.3 & $59.7(37.9)$ \\
\hline & 2025 & 41.2 & 105.0 & 41.1 & -10.6 & 151.5 & 153.9 & 119.2 & 143.1 & 129.8 & 95.5 & 100.4 & $97.3(52.7)$ \\
\hline \multicolumn{14}{|c|}{ Probability of transmission on ART $=0.5 \%$ female to male. ART enrolment begins in stage 2 at rate $0.9 /$ year (figure $2 \mathrm{E}$ ) } \\
\hline \multicolumn{14}{|c|}{ Change partner turnover rate } \\
\hline \multirow[t]{2}{*}{ Increase by $12.5 \%$} & 2015 & 10.3 & 6.4 & 7.1 & 9.6 & 10.9 & 8.8 & 7.5 & 8.5 & 6.2 & 6.6 & 6.0 & $8.0(1.7)$ \\
\hline & 2025 & 14.5 & 6.3 & 10.0 & 16.2 & 14.7 & 10.4 & 8.0 & 9.9 & 6.5 & 9.2 & 5.5 & $10.1(3.6)$ \\
\hline Increase by $50 \%$ & 2015 & 43.2 & 19.9 & 32.9 & 65.6 & 40.9 & 34.4 & 25.0 & 31.0 & 24.0 & 30.9 & 20.6 & $33.5(13.1)$ \\
\hline & 2025 & 58.7 & 19.2 & 45.7 & 110.0 & 48.0 & 36.1 & 25.4 & 33.9 & 24.1 & 43.2 & 18.6 & $42.1(25.9)$ \\
\hline Probability of transn & $2.0 \% \mathrm{fe}$ & ale to $\mathrm{m}$ & e. ART en & lment b & ns in sta & 2 at rate & 0.9/year ( & gure $2 \mathrm{~F}$ ) & & & & & \\
\hline Change partner turr & & & & & & & & & & & & & \\
\hline Increase by $12.5 \%$ & 2015 & 9.8 & 3.1 & 8.0 & 12.0 & 6.1 & 6.0 & 3.7 & 5.2 & 5.0 & 8.4 & 3.5 & $6.4(2.8)$ \\
\hline & 2025 & 12.0 & 2.9 & 9.5 & 18.7 & 6.0 & 6.2 & 3.9 & 5.1 & 4.9 & 10.5 & 3.6 & $7.6(4.7)$ \\
\hline Increase by $50 \%$ & 2015 & 41.6 & 11.6 & 35.1 & 71.1 & 24.8 & 24.8 & 14.4 & 20.6 & 20.7 & 38.7 & 12.9 & $28.8(17.4)$ \\
\hline & 2025 & 47.8 & 10.8 & 39.2 & 101.2 & 23.4 & 25.1 & 15.3 & 20.2 & 19.4 & 47.8 & 13.1 & $33.0(26.1)$ \\
\hline
\end{tabular}

*Though parameter value sets 3,10 , and 11 provided good model fits to empirically estimated prevalence, we believe they are less likely than the others because they estimated the HIV epidemic peaking too early (see figure 3 ).

ART, antiretroviral therapy.

In the extreme scenario in which ART was initiated beginning during HIV stage 1, HIV transmission probability on ART female to male was $0.5 \%$, and there was no behaviour change, the estimated impacts from the 11 parameter sets were inconsistent. Three of the sets suggested a drop in HIV prevalence by 2025, while eight suggested an increase. Across all parameter sets, the mean change in HIV prevalence over the scenario of no ART was a $24.7 \%$ rise assuming that the rate of ART enrolment was $0.25 /$ year and a $9.8 \%$ rise if the rate of ART enrolment was $1.00 /$ year (table 2 , scenario 3 ). If the probability of transmission while on ART was $2 \%$ female to male, all but one of the modelled parameter sets indicated that ART would result in rising HIV prevalence (table 2, scenario 4).

Not surprisingly, all sets of parameter values suggested that an increase in partner turnover rate would result in rising HIV prevalence. For example, if the probability of HIV transmission while on ART was just $0.5 \%$ female to male, and ART enrolment began in stage 2 at a very high rate of $0.9 /$ year, then the mean increase in prevalence by 2025 caused by a $12.5 \%$ increase in partner turnover rate was $10.1 \%$ (table 2, scenario 5).

\section{Incidence}

Without behaviour change, ART reduces HIV incidence (figure 4). This assumes a high rate of ART enrolment beginning in HIV stage 2, and that ART lowers the probability of HIV infection per partnership to $0.5 \%$ female to male and $1 \%$ male to female. Without behaviour change, our model suggests that ART would lower incidence from 14.7 to 8.9 per 1000 person-years observation (pyo) among men, and from 19.3 to 13.7 among women. If average partner turnover rate increases by $12.5 \%$, then our results indicate that the dual impact of ART and behaviour change would lower incidence to 10.3 per 1000 pyo among men, and to 15.3 among women. If partner turnover rate increases by $50 \%$, then incidence would initially rise, but would then stabilise at approximately the same values as without ART. 
Figure 4 Incidence among age 15-54 antiretroviral therapy (ART) initiation rate $=0.9 /$ year beginning in HIV stage 2. Rate of movement from high to low mortality on $A R T=0.5 /$ year.
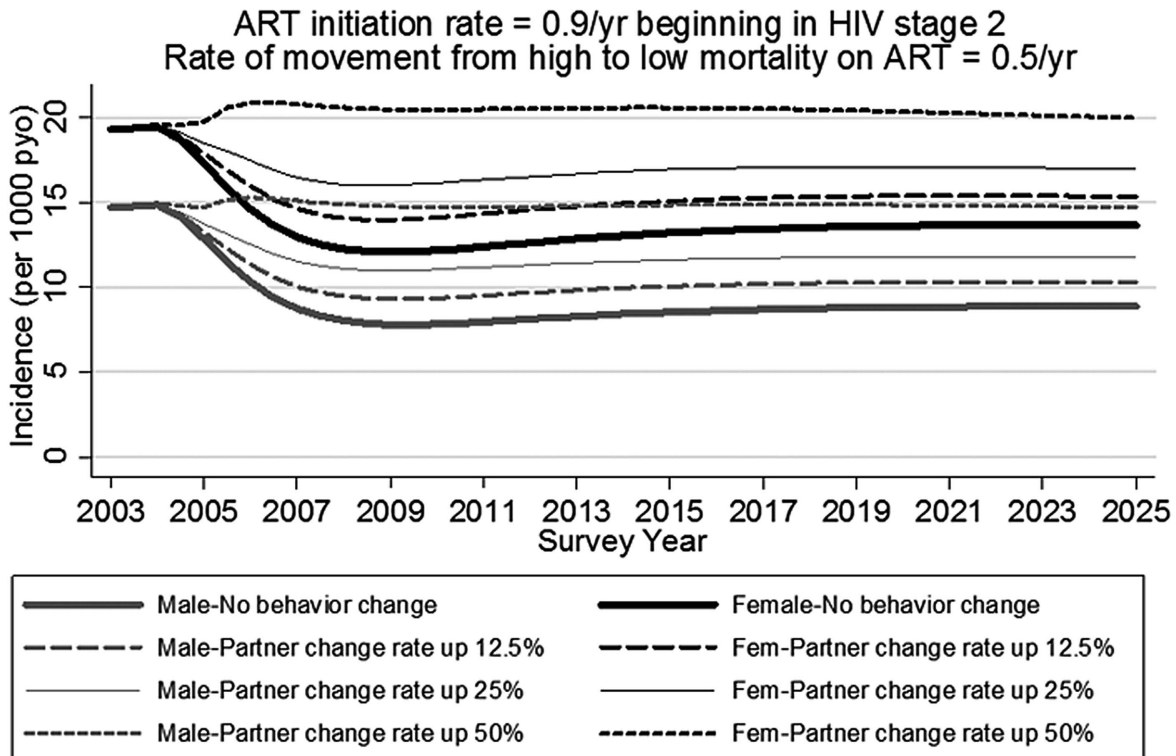

In the figure 4 scenario, with no behaviour change, $18.4 \%$ of incidence by 2025 comes from people in stage 1, 6.6\% from people in stage 2 , and $73.4 \%$ from people on ART. Just $1.6 \%$ of incidence comes from people in stages 3 and 4. By contrast, in the same scenario but in the year prior to ART introduction, $36.2 \%$ of incidence comes from people in stage 1, 40.3\% from stage $2,16.5 \%$ from stage 3 and $7.0 \%$ from stage 4 . The lower percent of incidence in stage 4 despite high infectiousness results from: a reduction in partner turnover rate in stage 4 (see online supplementary table S1), and mortality before stage 4 such that some people never reach this stage.

\section{DISCUSSION \\ Epidemiologic forecast}

Recent evidence from clinical trials have shown the positive impact that ART may have on reducing new infections (incidence). ${ }^{2}$ Our modelling work supports this evidence by also showing a reduction in incidence, unless partner turnover rates increase greatly $(>50 \%)$. However, although incidence falls, results suggested that HIV prevalence will be higher with ART than without. Among the scenarios examined, HIV prevalence may decrease among men only in an extreme case which may not be achievable in practice. That is, ART enrolment would need to begin while still in HIV stage 1, the rate of ART enrolment would need to be greater than 0.50 /year, sexual partner turnover would not change, and the probability of transmission while on ART would be just $0.5 \%$ per partnership from female to male (representing $>95 \%$ reduction in transmission compared to no ART). Even in this case, 8 of the 11 sets of parameter values that we assessed suggested that HIV prevalence would be higher in the presence of ART than in its absence.

Despite the short duration in the initial HIV stage of infection, our model estimates that in the absence of ART about 36\% of incidence comes from partnerships formed in which the HIV-infected partner was in the highly infectious stage 1. Initiating ART during this stage, if feasible, would therefore have the greatest impact on the epidemic.

Previous studies have suggested a range of potential outcomes to rolling out ART, from increasing ${ }^{6}{ }^{13}$ to decreasing HIV

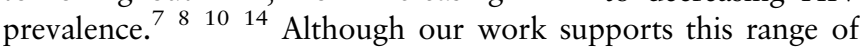
potential outcomes to rolling out ART, in all but the most extreme scenarios our results consistently suggest that HIV prevalence will rise as a result of ART.

Results from this study come from a model that was parameterised and fit using data from Uganda. We expect that our results provide a good estimate of the impact of ART and corresponding sexual behaviour change on HIV epidemiologic trends in countries that are similar to Uganda in terms of HIV prevalence trends pre-ART and HIV modes of transmission (mainly heterosexual sex).

\section{Limitations}

Results of any modelling study may be influenced by model assumptions. We have described our model assumptions in detail in the online supplementary appendix. One model assumption was that of no HIV/AIDS-related mortality after the initial period on ART. This is likely overly optimistic as nonperfect ART adherence speeds HIV progression and a higher mortality rate. If we had modelled a slightly higher mortality rate, even among those on ART, then estimated HIV prevalence after ART is introduced would be lower, as infected people would die at a higher rate. Also, we used a deterministic mathematical model. As such, stochastic events that may influence the plausible range of the impact of ART on HIV prevalence and incidence were not modelled.

In our best fitting model simulation, $36.2 \%$ of HIV incidence prior to ART introduction came from people in HIV stage 1. While this is similar to that estimated elsewhere, ${ }^{31} 32$ it is higher than that estimated by some. ${ }^{33}$ If more HIV incidence is attributable to higher HIV stages than we estimated, then ART enrolment in later stages would have a greater impact on incidence and subsequent prevalence.

\section{CONCLUSION}

It is unlikely that most HIV infections could be identified during the high viraemic stage 1 of infection. If infections are identified at a rate of $0.9 /$ year beginning in stage 2 , and ART is initiated immediately after detection, our results indicate that HIV incidence would fall as a result of ART. However, this comes at a cost. Our results still indicate rising prevalence as those infected live longer. The combined effect of increased risky sexual behaviour and longer life expectancy while infected would increase HIV prevalence dramatically. Care providers should be prepared 
for a higher than previously suggested ${ }^{11}$ number of people who are likely to need treatment.

Key messages

- Although HIV incidence will fall, HIV prevalence will rise as a result of antiretroviral therapy.

- If the possible increase in risky sexual behaviour is not controlled, HIV prevalence will rise even further.

- Policy makers must prepare to provide care for the increased population living with HIV.

- Policy makers are urged to continue HIV prevention activities, including promoting sex education.

\section{Handling editor Jackie A Cassell}

Contributors LAS, RNN, RGW, KO and RC developed the model. LAS fit the model and wrote the manuscript. LAS, RNN and RC conducted literature review. BNM directed the MRC cohort from which data was provided. All authors contributed suggestions to the manuscript.

Funding This work was supported by the Medical Research Council-UK, grant number G0501499. RGW is funded by the Medical Research Council (UK) (MR/J005088/1 and G0802414), the Bill and Melinda Gates Foundation (TB Modelling and Analysis Consortium: Grants 21675 / OPP1084276 and Consortium to Respond Effectively to the AIDS/TB Epidemic: 19790.01), and CDC/PEPFAR via the Aurum Institute (U2GPS0008111). The funders had no involvement in the design, collection, analysis or interpretation of the data, in writing the report or in the decision to submit.

Competing interests None.

Ethics approval This study was approved by the Science and Ethics Committee of the Uganda Virus Research Institute.

Provenance and peer review Not commissioned; externally peer reviewed.

Data sharing statement Data used to inform our mathematical model came from a 21-year cohort in rural Uganda. This is a sero-behavioral general population cohort, comprising 25 villages. The Medical Research Council in Uganda has data on numerous sexual behaviour indicators, collected annually from each member of the cohort, as well as annual HIV test results.

Open Access This is an Open Access article distributed in accordance with the terms of the Creative Commons Attribution (CC BY 3.0) license, which permits others to distribute, remix, adapt and build upon this work, for commercial use, provided the original work is properly cited. See: http://creativecommons.org/ licenses/by/3.0/

\section{REFERENCES}

1 Ford N, Calmy A, Mills EJ. The first decade of antiretroviral therapy in Africa. Global Health 2011;7:33.

2 Cohen MS, Chen YQ, McCauley M, et al. Prevention of HIV-1 infection with early antiretroviral therapy. N Engl J Med 2011;365:493-505.

3 Abbas UL, Mellors JW. Interruption of antiretroviral therapy to augment immune control of chronic HIV-1 infection: risk without reward. Proc Natl Acad Sci USA 2002;99:13377-8.

4 Nagelkerke N, Jha P, De Vlas $\mathrm{S}$, et al. Modelling HIVIAIDS epidemics in Botswana and India: impact of interventions to prevent transmission. Bull World Health Organ 2002;80:89-96.

5 Law M, Prestage G, Grulich A, et al. Modelling the effect of combination antiretroviral treatments on HIV incidence. AIDS 2001;15:1287-94.

6 Gray R, Xianbin L, Wawer M, et al. Stochastic simulation of the impact of antiretroviral therapy and HIV vaccines on HIV transmission; Rakai, Uganda. AIDS 2003;17:1941-51.
7 Blower S, Bodine E, Kahn J, et al. The antiretroviral rollout and drug-resistant HIV in Africa: insights from empirical data and theoretical models. AIDS 2005;19: $1-14$.

8 Blower S, Ma L, Farmer P, et al. Predicting the impact of antiretrovirals in resource-poor settings: prevenging HIV infections whilst controlling drug resistance. Curr Drug Targets Infect Discord 2003;3:345-53.

9 Baggaley RF, Garnett GP, Ferguson NM. Modelling the impact of antiretroviral use in resource-poor settings. PLoS Med 2006;3:e124.

10 Velasco-Hernandez J, Gershengorn H, Blow S. Could widespread use of combination antiretroviral therapy eradicate HIV epidemics? Lancet Infect Dis 2002;2:487-93.

11 Granich R, Gilks C, Dye C, et al. Universal voluntary HIV testing with immediate antiretroviral therapy as a strategy for elimination of HIV transmission: a mathematical model. Lancet 2009;373:48-57.

12 Dodd P, Garnett G, Hallett T. Examining the promise of HIV elimination by 'test and treat' in hyperendemic settings. AIDS 2010;24:729-35.

13 Baggaley RF, Ferguson NM, Garnett GP. The epidemiological impact of antiretroviral use predicted by mathematical models: a review. Emerg Themes Epidemiol 2005:2:9.

14 Granich RM, Gilks CF, Dye C, et al. Universal voluntary HIV testing with immediate antiretroviral therapy as a strategy for elimination of HIV transmission: a mathematical model. Lancet 2009;373:48-57.

15 Cohen M. HIV Transmission Risk Under ART. XVII International AIDS Conference. 2008.

16 Temoshok L, Wald R. Integrating multidimensional HIV prevention programs into healthcare settings. Psychosematic Med 2008;70:612-19.

17 Porco T, Martin J, Page-Shafer K, et al. Decline in HIV infectivity following the introduction of highly active antiretroviral therapy. AIDS 2004;18:81-8.

18 Scheer S, Chu P, Klausner J, et al. Effect of highly active antiretroviral therapy on diagnoses of sexually transmitted diseases in people with AIDS. Lancet 2001;357:432-5

19 Olley B. Higher-risk sexual behaviour among HIV patients receiving antiretroviral treatment in Ibadan, Nigeria. African J AIDS Res 2008;7:71-8.

20 Bunnell R, Ekwaru J, Solberg P, et al. Changes in sexual behavior and risk of HIV transmission after antiretroviral therapy and prevention interventions in Uganda. AIDS 2006;20:85-92.

21 Shafer L, Nsubuga R, White R, et al. Antiretroviral therapy and sexual behavior in Uganda: a cohort study. AIDS 2011;25:671-8.

22 Mulder D, Nunn A, Kamali A, et al. Two-year HIV-1-associated mortality in a Ugandan rural population. Lancet 1994;343:1021-3.

23 Seeley J, Wagner U, Mulemwa J, et al. The development of a community-based HIVIAIDS counselling service in a rural area in Uganda. AIDS Care 1991;3: 207-17.

24 Seeley J, Malamba S, Nunn A, et al. Socioeconomic Status, Gender, Risk of HIV-1 Infection in a Rural Community in South West Uganda. Med Anthrop Q 1994;8: 78-89.

25 Mbulaiteye S, Mahe C, Whitworth J, et al. Declining HIV-1 incidence and associated prevalence over 10 years in a rural population in south-west Uganda: a cohort study. Lancet 2002;360:41-6.

26 Shafer L, Biraro S, Nakiyingi-Miiro J, et al. HIV Prevalence and Incidence are no longer falling in Southwest Uganda: evidence from a Rural Population Cohort 1989-2005. AIDS 2008;22:1641-49.

27 Kamali A, Carpenter LM, Whitworth JAG, et al. Seven-year trends in HIV-1 infection rates, and changes in sexual behaviour, among adults in rural Uganda. AIDS 2000;14:427.

28 Weinstein M. Recent developments in decision-analytic modelling for economic evaluation. Pharmacoeconomics 2006:24:1043-53.

29 Kim J, Kuntz K, Stout N, et al. Multiparameter Calibration of a Natural History Model of Cervical Cancer. Amer J Epidemiol 2007;166:137-50.

30 Keeling M, Rohani P. Modeling infectious diseases in humans and animals. 1st edn. Princeton: Princeton University Press, 2007.

31 Powers K, Poole C, Pettifor A, et al. Rethinking the heterosexual infectivity of HIV-1: a systematic review and meta-analysis. Lancet Infect Dis 2008;8:553-63.

32 Powers KA, Ghani AC, Miller WC, et al. The role of acute and early HIV infection in the spread of HIV and implications for transmission prevention strategies in Lilongwe, Malawi: a modelling study. Lancet 2011;378:256-68.

33 Xiridou M, Geskus R, de Wit J, et al. Primary HIV infection as source of HIV transmission within steady and casual partnerships among homosexual men. AIDS 2004;18:1311-20. 